\title{
The data acquisition and trigger system of the Belle II experiment
}

\author{
Chunhua Li*i+ \\ The University of Melbourne \\ E-mail: chunhua.li@unimelb.edu.au
}

The Belle II experiment operating at SuperKEKB, a electron-positron collider in Japan, is a new particle physics experiment, that will perform a new panorama of measurements in heavy flavor physics and provide an important and unique source of information on the details of the physics beyond the standard model (SM). The designed instantaneous luminosity of SuperKEKB is $8 \times$ $10^{35} \mathrm{~cm}^{-2} \mathrm{~s}^{-1}$, meaning that the collider will produce tens of billions of interesting electronpositron collision events every year. The online Trigger and Data Acquisition (DAQ) systems are indispensable for Belle II to select physics-interesting events and transfer the data from front-end electronics to storage system.

A flexible and robust trigger system has been designed for Belle II. The main trigger are based on the central drift chamber (CDC) and the electromagetic calorimeter (ECL). Three-dimensional (3D) tracking on CDC trigger, Bhabha identification with 3D clusters information on ECL trigger, and low level reconstruction are being developed. These ensure that the events of interest are triggered with high efficiency.

The designed trigger rate of the DAQ system at Belle II is $30 \mathrm{kHz}$. To further suppress the event rate to $10 \mathrm{kHz}$ by rejecting the background, the software based high level trigger (HLT) is developed with full event reconstruction algorithms, which also provides the tracking information to the silicon pixel sensors (PXD) for its data size reduction. Parallel processing is applied on HLT due to the large data flow. More than 5 HLT units ( 320 cores per unit) provide strong computing power.

The European Physical Society Conference on High Energy Physics

22-29 July 2015

Vienna, Austria

* Speaker.

${ }^{\dagger}$ on behalf of Belle II collaboration 


\section{Introduction}

SuperKEKB is an electron-positron collider at KEK, Japan, with electron and positron beam energies of $7 \mathrm{GeV}$ and $4 \mathrm{GeV}$, respectively [1]. The Belle II experiment operating at SuperKEKB is a new particle physics experiment, which will perform a new panorama of measurements in heavy flavor physics and provide an important and unique source of information on the details of the physics beyond SM. The designed instantaneous luminosity of SuperKEKB is $8 \times 10^{35} \mathrm{~cm}^{-2} \mathrm{~s}^{-1}$, which is 40 times higher than the peak value of KEKB. The total cross sections and trigger rates of several physical processes of interest at the target luminosity are listed in Table 1.

Table 1: Toal cross section and trigger rate with luminosity of $8 \times 10^{35} \mathrm{~cm}^{-2} \mathrm{~s}^{-1}$ at the $\Upsilon(4 S)$.

\begin{tabular}{lcc}
\hline Physics process & Cross section $(\mathrm{nb})$ & Rate $(\mathrm{Hz})$ \\
\hline$B \bar{B}$ & 1.2 & 960 \\
continuum & 2.8 & 2200 \\
$\mu \mu$ & 0.8 & 640 \\
$\tau \tau$ & 0.8 & 640 \\
Bhabha & 44 & $450^{*}$ \\
$\gamma \gamma$ & 2.4 & $19^{*}$ \\
Two photon & 13 & $10000^{* *}$ \\
\hline Total & 67 & 15000 \\
\hline$*$ Rates of Bhabha and $\gamma \gamma$ are pre-scaled by factor of 100. \\
** Rate estimated from the Belle L1 trigger.
\end{tabular}

SuperKEKB applies Nano-Beam scheme, which was first proposed for the Super B factory in Italy [2], to increase the instantaneous luminosity by squeezing the beam size. Touschek scattering is intra-bunch scattering. It changes the momenta of beam particles so that they hit the vacuum chamber and magnet wall. This background is proportional to the beam bunch current, the number of bunches, and the inverse of the beam size. Therefore the Touscheck background is expected to be the major background source with 20-30 times larger than KEKB.

Since the $B \bar{B}$ and continuum processes have high multiplicity in the final states which is much different from the backgrounds, they are easy to be triggered by requiring a large number of tracks in events. Many physics processes e.g. $\tau$ 's leptonic decays and dark matter search, however, have zero or two tracks only in their final states, it is difficult to be separated from the backgrounds. In addition, the topologies of some low multiplicity processes are similar to that of Bhabha which has a huge cross section as listed in Table 1. It is necessary to veto Bhabha in trigger to suppress the event rate, which will lose the trigger efficiencies of the low multiplicity. To keep high efficiencies of the processes of interest, an effective trigger system is essential.

\section{Trigger}

The trigger system contains the hardware based Level 1 (L1) trigger and the software based High Level Trigger (HLT) which will be described in DAQ section as a component of DAQ. 


\subsection{Trigger Requirements}

The function of the L1 trigger is to select the events of interest including $B \bar{B}$, continuum, taupair, and low multiplicity processes by using a set of triggers (menu), and reject the backgrounds. Some event types with loose trigger criteria are prescaled for the trigger efficiency, detector and backgorund studies. The trigger efficiencies of hadronic events from $Y(4 S) \rightarrow B \bar{B}$ and continuum should be larger than $99.9 \%$, and for low multiplicity processes be high. The event rate of the physics process is $15 \mathrm{kHz}$ as listed in Table 1 . The total trigger rate has to be less than $30 \mathrm{kHz}$ which is the designed maximum trigger rate of DAQ. The latency of the L1 trigger is about $5 \mu s$ [3]. The trigger must be flexible and robust in order to function even under extreme background situations.

\subsection{L1 Trigger System}

To meet these requirements, Belle II adopts the same triggering scheme as Belle with new technologies [4]. The trigger system consists of sub-trigger systems, one global reconstruction logic (GRL) and one final decision logic (GDL) as shown in Figure 1. The sub-detector information and sub-trigger results are sent to GRL where a low level reconstruction is performed by combining these information. The reoncstruction results are sent to GDL which then issues a trigger based on these detector information.

In Belle II, all components of trigger system are replaced with new technologies. Each component has a Field Programmable Gate Array (FPGA) so that the trigger logic is configurable rather than hard-wired. All data flow along high speed serial links, which enables us to funnel a huge amount of information to one FPGA.

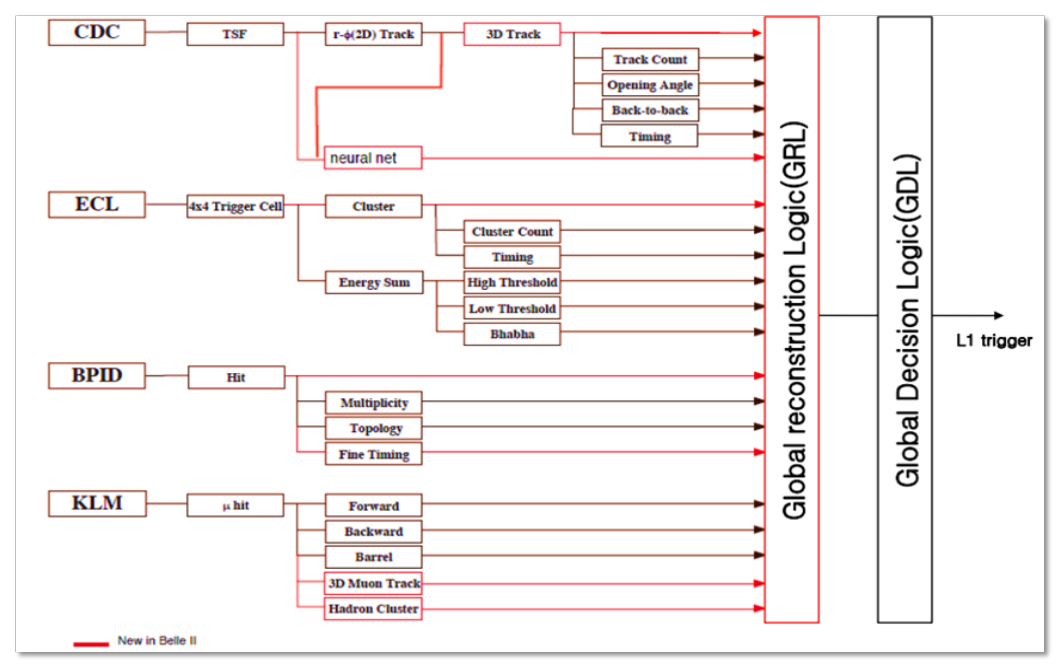

Figure 1: The L1 trigger scheme. The red lines indicate the new components increased in Belle II.

The higher background and low multipliticy program challenges the trigger system. In order to keep high efficiency of the events of interest and reject background effectively, many significant improvements in the trigger system are being developed. The main L1 triggers are based on the central drift chamber (CDC) and the electromagnetic calorimeter (ECL) [3]. 
The CDC sub-trigger finds and characterizes the charged tracks detected by the drift chamber. The new CDC contains about 50,000 sense and field wires. The sense wires are arranged in layers, where 6 or 8 adjacent layers are combined in a SuperLayer (SL). The SL alternate between axial orientation, aligned with the solenoidal magnetic field (z-axis), and stereo orientation. The strategy of the present CDC track trigger is based on so-called track segments (TS), which are produced for each of the 9 SL by the Track Segment Finder. The 2D trigger follows the strategy of the previous Belle CDC trigger. The 2D tracks in $r-\phi$ space is reconstructed by combining TS from the SL with axial wire orientation.

Touschek scattering produces a high rate of background events with the longitudinal position of the vertex (z-vertex) outside of the nominal interaction region, where the physically interesting reactions from the $e^{+} e^{-}$collisions are produced. By reconstructing the z-vertex within the latency of L1 trigger, a large fraction of this background can be suppressed. By combining the information of axial and stereo wires it is possible to reconstruct a full $3 \mathrm{D}$ helix track, which can be parametrized by $(p T, \phi, \theta, z, d)$, where $p T$ is the transverse momentum, $\phi$ and $\theta$ are the azimuthal and polar angle at the vertex, $z$ is the vertex position along the beamline and $d$ is the displacement of the vertex from the beamline in the $r-\phi$ plane. The total latency of $5 \mu s$ for the full L1 trigger allows for only $\approx 1 \mu s$ for the z-vertex reconstruction. Neural networks [5] of the Multi Layer Perceptron (MLP) type are well suited to meet these constraints due to their inherent parallelism and deterministic runtime.The proposed neural trigger will run in parallel to the conventional analytical 3D trigger and employ MLPs trained on sample tracks to predict the z-vertex.

The calorimeter can generate fast trigger signals that provide a fully efficient trigger for both neutral and charged particle oriented physics events. Two complimentary trigger schemes are taken into account: a total energy trigger and an isolated cluster counting trigger. The former is sensitive to physics events with high electromagnetic energy deposition while the latter is sensitive to multi-hadronic physics events that have low-energy clusters and/or minimum ionizing particles. In addition, the ECL trigger system could also identify Bhabha and $\gamma \gamma$ events for online luminosity measurement and data taking monitoring. The logic of Bhabha and $\gamma \gamma$ requires two clusters with high energy which must satisfy back-to-back topology using a look-up table. This logic can identify Bhabha and $\gamma \gamma$ events with high purity, and is essential to keep the high trigger efficiency of low multiplicity processes.

Another important improvement of the Belle II trigger system is the new GRL. Instead of sending the sub-trigger information to GDL directly, low level reconstruction is performed by using the sub-detector information, such as the match between CDC and ECL in the GRL. The GRL provides more useful information for trigger, and improves the trigger performance significantly.

\section{DAQ}

The DAQ system reads out detector signals from the frontend electronics to the storage system upon the L1 trigger decision. The main components of the Belle II DAQ system are the unified data link called the Belle2Link, the common readout platform called COPPER, the event builder system, and the HLT system as shown in Figure 2. The detector front-end boards with digitizers are placed near or inside the detector structure and the digitized signals are transferred into COPPER systems through long optical fibers using the Belle2Link. A simple data reduction is performed on each 
front-end electronics board or on the receiver module of COPPER, while the data formatting and module-level event building is done on COPPER using the on-board CPU. Further event building and reduction are done on the readout PCs and the event builder, and finally processed by the HLT farms for the software event selection.

The nominal average $\mathrm{L} 1$ trigger rate is expected to be $15 \mathrm{kHz}$ at the instantaneous luminosity of $8 \times 10^{35} \mathrm{~cm}^{-2} \mathrm{~s}^{-1}$ as listed in Table 1 . The designed event rate of DAQ is $30 \mathrm{kHz}$. Since the expected event size from the silicon pixel sensors (PXD) is very large ( $1 \mathrm{MB} /$ event), it is quite difficult to manage its readout using COPPERs, so an additional data flow is designed for PXD data readout. The event size before HLT is estimated to be $100 \mathrm{kB}$.

Due to the limitation of storage space, the software trigger HLT is being developed to suppress the backgrounds further. The HLT contains two components: a Level 3 (L3) trigger and a physics trigger. The role of L3 is to reduce the load of physics trigger. By using dedicated fast reconstruction algorithms with CDC and ECL information, the event rate is suppressed to be $15 \mathrm{kHz}$ from $30 \mathrm{kHz}$ by L3 trigger. The events passing L3 trigger are reconstructed with the offline reconstruction algorithm with all detectors information except PXD. Based on the reconstruction infomation, physics trigger classifies the events to kinds of categories e.g. $B \bar{B}$, tau-pair. The event rate is further suppressed to be $10 \mathrm{kHz}$. The beam-induced backgrounds are expected to be high at the first period of data taking, and some selection criteria also need to be changed according to the different center of mass energies and physics motivations, so a trigger menu is being developed to face these conditions. The output event size of HLT is $200 \mathrm{kB}$. In addition, the processing result of the HLT, containing the event tag and parameters of the tracks in the event, are sent to the ATCA boards (or PCs) which receive the data from PXD. These information is used for the calculation of Region of Interest (RoI) of PXD. The selected PXD hits are sent to the second-level event builder to combine with the data from other detectors.

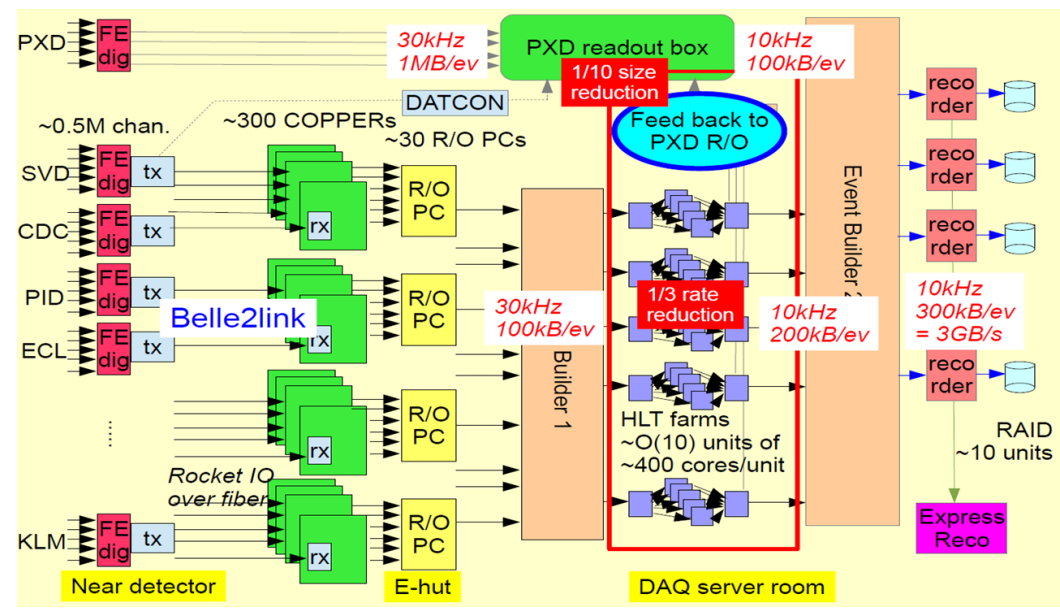

Figure 2: The structure of the DAQ system.

Parallel processing of computing, supported by basf2 (the Belle II software framework), is essential in the HLT due to the large data flow. There are more than five HLT units ( $\sim 320$ cores per unit) at the instantaneous luminosity $2 \times 10^{35} \mathrm{~cm}^{-2} \mathrm{~s}^{-1}$. The number of units will be added as 
the increase of luminosity. The powerful computing source ensures that the HLT processes the data fast.

Cosmic ray tests of sub-detectors and DAQ have been performed since November, 2014. The system of ECL and CDC data taking were run successfully.

\section{Summary}

The trigger and DAQ systems of Belle II have had many improvements compared with Belle. 3D tracking on CDC trigger, Bhabha identification with 3D clusters information on ECL trigger, and low level reconstruction are developed, the software trigger with full reconstruction algorithm is performed. These make sure that the events of interest are triggered with high efficiency. Belle II commissioning without vertex detectors is on May 2017, the trigger and DAQ systems will be ready by then.

\section{References}

[1] T. Abe, Belle II Technical Design Report, arXiv: 1011.0352.

[2] P. Raimondi, talk given at the 2nd SuperB workshop, Frascati, http://www.lnf.infn.it/conference/superb06/talks/raimondi1.ppt, 2006.

[3] Y. Iwasaki et al., IEEE transactions on nuclear science, VOL. 58, NO. 4, (2011)

[4] A. Abashian et al., Belle Collaboration, "The Belle detector", Nucl. Instrum. Methods A, vol. 479, pp. 117-232, 2002.

[5] S. Neuhaus et al., A neural network z-vertex trigger for Belle II, arXiv:1410.1395. 